\title{
Influence of Surface Modifying Macromolecules (SMMs) on Polyethersulfone Hollow Fiber Membranes for Water Separations
}

\author{
N. Bolong ${ }^{1}$, A. F. Ismail ${ }^{2 *}$, M. R. Salim ${ }^{3}$, D. Rana ${ }^{4}$ \& T. Matsuura ${ }^{5}$ \\ ${ }^{1 \& 2}$ Advanced Membrane Technology Research Centre, Faculty of Chemical \& Natural Resources Engineering, \\ Universiti Teknologi Malaysia, 81310 Skudai, Johor, Malaysia \\ ${ }^{1 \& 3}$ Dept. of Environmental Engineering, Faculty of Civil Engineering, Universiti Teknologi Malaysia, 81310 \\ Skudai, Johor Malaysia \\ ${ }^{1}$ Civil Engineering Programme, School of Engineering \& IT, Universiti Malaysia Sabah, 88999 Kota Kinabalu, \\ Sabah, Malaysia \\ ${ }^{4} \& 5$ Industrial Membrane Research Laboratory, Dept. of Chemical and Biological Engineering, Univ. of Ottawa, \\ 161 Louis Pasteur St, P.O Box 450, Stn A, Ottawa, Ont. Canada K1N 6N5
}

\begin{abstract}
The objective of this work is to modify the polyethersulfone (PES) hollow fiber membranes by blending with Surface Modifying Macromolecules (SMMs) which were able to introduce charges at the membrane surface. To study the effects of SMMs, PES solutions with and without blending SMMs were spun by the dry-wet phase inversion method. The membranes were characterized by ionic solutes permeation and Field Emission Scanning Electron Microscopy (FESEM). There was no significant difference in the membrane morphology between the membranes with and without SMM blending, indicating that the morphology of the base polymer remained relatively unchanged. However, based on Energy Dispersive X-ray (EDX) analysis, the presence of SMM's elements at the surface of the SMM blended membranes was detected. The pure water permeation rates were found two times increased with the existence of SMMs, while rejection towards ionic solutes remained relatively unchanged.
\end{abstract}

Keywords: Charged-Surface Modifying Macromolecules (SMMs), polyethersulfone hollow fiber membranes, ionic separations

\subsection{INTRODUCTION}

One of the most promising options for pollutants separation and purification, according to Thomas [1], is membrane technology. Membrane processes have become more popular in water treatment because the processes can disinfect water without chemical additions and avoid the formation of toxic disinfection byproducts $[2,3]$. Moreover, membrane technology has received more interest in recent years due to the stringent standard for water supply and effluent discharge. The benefits of membrane treatment processes

\footnotetext{
*Correspondence to: A. F. Ismail (email: afauzi@utm.my)
}

has been highlighted [3-5] as; compact, modular construction, minimum of moving parts with low maintenance requirements, no chemical addition requirements and minimal chemical sludge disposal, absolute barriers to particles and pathogens, constant filtered water quality irrespective of feed water quality, easy system upgrading and suitability to small systems and distributed locations. In membrane processes, the success of the separation system depends on the quality and suitability of the membrane that is incorporated into the system. The properties of the membranes such as permeability, separation capability etc. specific for required application would be crucial for a successful separation 
system. Hence, in membrane development, one important goal is to optimize and control membrane properties and structures to achieve the required performance.

In the preparation of asymmetric membranes by the phase inversion technique, several variables can be adjusted to optimize the membrane properties. One of the potential techniques to control membrane performance is by membrane surface modification. There are several techniques used for membrane surface modification, including cross-linking, grafting, preadsorption of water-soluble polymers etc. [6-8]. However, the modification technique via blending surface modifying macromolecules (SMMs) to polymer casting solutions has better advantage as it can produce surface-modified membranes via a single-step casting procedure $[2,9]$. Blending of poly (vinly pyrrolidone) (PVP) as a second polymer has demonstrated to 'hydrophilized' polysulfone (PS) or polyethersulfone (PES) and also tune pore structure; and yet the modification is not permanent as a fraction of the PVP will be washed out during usage [9].

Flat membrane formation in which functional macromolecules were blended as surface active amphiphilic block copolymers was studied by several researchers $[2,10-14]$.Their studies found that the mixing of the compatible blocks with the matrix polymer led to an efficient anchoring, while the surface segregation of the functional blocks led to a modified membrane surface.

The main feature of SMMs is the low surface free energy than that of the base polymer. As a result, it has the ability to migrate preferentially toward a polymer-air interface [12]. In the case of hydrophobic SMMs, migration makes the surface significantly more hydrophobic with low surface energy [10-14]. It has been shown that SMMs migrated to the surface and the hydrophobic fluoro-hydrocarbon end groups oriented themselves toward the air-polymer interface, reducing the surface energy of the hydrophilic base polymer to values close to that of polytetrafluoroethylene (Teflon) [11]. Since only a small amount of SMMs was used, the bulk properties of the base polymer remained relatively unchanged. The concept was later extended to hydrophilic SMMs [2].
It is attempted to extend the concept of SMMs further to charged surface-modifying macromolecules (cSMMs). cSMMs are oligomeric glycol polymers synthesized by polyurethane chemistry and tailored with end groups that carry sulfonate ions.

In this study, polyethersulfone (PES) was used as the base polymer. PES is a closely related derivative of polysulfone (PS) and has a high glass transition temperature of $230^{\circ} \mathrm{C}$. As membrane material, it has the advantages such as good membrane forming properties, high mechanical and chemical stability, and is commercially available and relatively inexpensive. An attempt to modify the PES hollow fiber membrane by blending with charged surface modifying macromolecules (CSMM) was the focus of this study. Hypothetically, the charges were contributed by the end-capped group of cSMM namely hydroxybenzene sulfonate (HBS). Consequently, the objectives of the current research are to test the miscibility of CSMM in SEM, to fabricate PES hollow fibers blended with cSMMs and to study the hollow fiber morphology. Then, the ionic salt separation performance of the PES membranes with and without cSMM blending is evaluated.

\subsection{EXPERIMENTAL METHODS}

\subsection{Materials}

Polyethersulfone (PES) RADEL A-300 with a molecular weight of about 15,000 Da was purchased from Amoco Chemicals to be used as the base membrane material. N-methyl-2pyrrolidinone (NMP, >99\%) supplied by MERCK, Darmstadt, Germany was used as a solvent. Water was used as a coagulation medium. Sodium chloride $(\mathrm{NaCl})$ of analytical grade supplied by Merck, Darmstadt, Germany was used as received.

The cSMMs used for dope formulation in this work were laboratory synthesized and have the structures shown in Figure 1. Based on the softsegment of the polyurethane prepolymer that is polyethylene glycol (PEG) with end capping group, they are called poly(ethylene glycol)Hydroxybenzene sulfonate (PEG-HBS). 


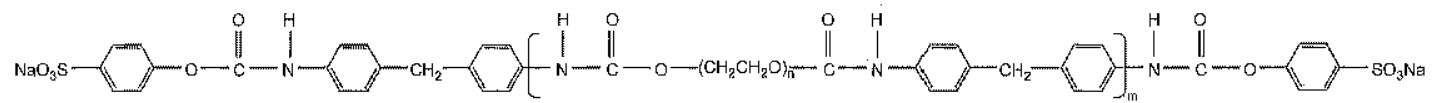

Figure 1 Chemical structures of Poly (ethylene glycol)-4,4' methylene bis (phenyl isocyannateHydroxybenzene sulfonate (PEG-HBS)

\subsection{Dope Solution Preparation and Hollow Fiber Spinning}

Two types of polymer solutions namely PES/NMP $(22 / 78)$ and PES/NMP/PEG-HBS (22/77/1) were prepared. The concentration of 22 weight percent of PES was used as it is suitable for the fabrication towards high performance nanofiltration [15]. Only $1 \mathrm{w} \%$ of CSMM was added to avoid massive changes to the base material. Moreover, it was reported that only a small amount of SMM was needed to cover the membrane surfaces $[2,16]$. The PES powder was initially dried in a vacuum oven at $120^{\circ} \mathrm{C}$ for $5 \mathrm{~h}$ to remove its moisture content. Then, the dried PES was dispersed slowly into the solvent NMP or the mixture of NMP/CSMM within a time frame of 30-45 minutes at $40^{\circ} \mathrm{C}$ and kept in a flask under a high-speed mechanical stirring.

Then, the mixture was slowly stirred at room temperature for $8 \mathrm{~h}$ in order to obtain a homogenous solution. The solution was then ultrasonicated for about $2 \mathrm{~h}$ to remove the bubbles and then kept at room temperature for $24 \mathrm{~h}$ prior to the spinning process.

Hollow fiber membranes were spun using the dry-jet wet spinning process as described in our previous work [17]. The phase inversion method was used to prepare the membranes. The formulated dope was fed into the annulus of the spinneret under pressurized (1-1.5 psig) nitrogen gas. The polymer dope was extruded from the spinneret (spinneret dimensions: O.D. $600 \mu \mathrm{m}, \mathrm{I} . \mathrm{D} 300 \mu \mathrm{m}$ ) and spun without any extra drawing. This was to minimize the effect of spin line (drawing) or elongation stress on fiber formation at a high jet stretch ratio (ratio of drum take-up speed to dope extrusion speed). Water at slightly lower than the room temperature $\left(18-20^{\circ} \mathrm{C}\right)$ was used as the external coagulant to yield an outer selective dense layer fiber membrane.
After spinning, the as-spun fibers were rinsed with water at room temperature for at least 24 hours to remove the residual NMP. The fibers were then immersed in aqueous glycerol solution (50\%) for 24 hours to restrain the fiber's surface and to prevent rupture of the fiber structure. The hollow fibers were hung vertically and air-dried at room temperature for 7 days prior to the transfer to the test modules. The spinning process conditions of hollow fiber membranes are summarized in Table 1.

Table 1 Experimental parameters used in spinning hollow fibre membrane

\begin{tabular}{|c|c|}
\hline Parameter & $\begin{array}{l}\text { Value / process } \\
\text { conditions }\end{array}$ \\
\hline $\begin{array}{l}\text { Dope solution composition } \\
\text { (weight to weight ratio) }\end{array}$ & $\begin{array}{l}\text { PES/NMP (22/78) } \\
\text { PES/NMP/PEG- } \\
\text { HBS }(22 / 77 / 1)\end{array}$ \\
\hline Dope flow rate & $3.0 \mathrm{~cm}^{3} / \mathrm{min}$ \\
\hline Bore fluid composition & Water \\
\hline Bore fluid flow rate & $1.0 \mathrm{ml} / \mathrm{min}$ \\
\hline Coagulant temperature & $18^{\circ} \mathrm{C}$ \\
\hline Bore fluid temperature & $20 \pm 3^{\circ} \mathrm{C}$ \\
\hline Air gap & $\begin{array}{l}5 \mathrm{~cm} \text { (dry-wet } \\
\text { condition) }\end{array}$ \\
\hline
\end{tabular}

\subsection{Measurement of Permeation Flux and Rejection}

In order to test the hollow fiber separation performance in terms of permeation flux and sodium chloride rejection, permeation modules were prepared. Fibers with a length of $20 \pm 2 \mathrm{~cm}$ were assembled into bundles. The shell sides of the two ends of the bundles were glued into two stainless steel tees using a normal-setting epoxy resin. These modules were left overnight for curing before being tested. The permeation test 
was conducted by using a permeation testing-rig as described previously by [17]. The pressure normalized fluxes, $J_{w}\left(\mathrm{~L} / \mathrm{m}^{2}\right.$ h Bar $)$, of water through the hollow fiber modules were calculated using the following equation:

$$
J_{w}=\frac{V}{A t \Delta P}=\frac{V}{\pi n D L t \Delta P}
$$

Where $V$ is the permeate volume $(\mathrm{L}), t$ is the time of permeate collection (hour), $A$ is the effective surface area of the hollow fibre $\left(\mathrm{m}^{2}\right)$, $\Delta P$ is the trans-membrane pressure difference (Bar), $n$ is the number of tested fibers in the module, $D$ is the outer diameter of the fibers $(\mathrm{m})$ and $L$ is the effective length of the fibers $(\mathrm{m})$.

In this work, sodium chloride solution at concentration range of 0.01 to 0.03 molar was used as the feed solution. Both the feed and permeate solute concentration were measured using digital conductivity meter (WTW handheld meter model LF330). The membrane rejection percentage, $R \%$ is computed by equation 2 :

$$
R \%=\left(\frac{1-C_{p}}{C_{l}}\right) \times 100
$$

Where $C_{f}$ and $C_{p}$ are solute concentrations in feed and permeate, respectively.

In the filtration test system, the feed solution was pumped by a Hydra cell pump to the shell side of the membrane module at flow rates of $2.5 \pm 5$ to $\mathrm{L} / \mathrm{min}$. The pressure was controlled by a back pressure regulator and monitored by the pressure gauges installed at the feed inlet and the retentate outlet of the module. The permeate was collected from the lumen side whilst the concentrate was recycled back to the feed tank, Prior to the collection of the permeate sample, the hollow fibers were stabilized for 20-30 minutes by monitoring the permeation rate consistency. Each test was repeated three times and the average values are reported.

\subsection{Field Emission Scanning Electron Microscopy (FESEM) Analysis}

Scanning electron microscopy (SUPRA FESEM) was used to study the morphology of the hollow fiber nembranes. The elemental compositions of the hollow fiber membrane surface were deter- mined by energy dispersive $\mathrm{x}$-ray analysis facility which is attached to the FESEM. In order to assess the cross-section, fiber samples were inmersed in liquid nitrogen for approximately 10 seconds and fractured to get a generally clean break. Each hollow fiber cross-section was carefully mounted on a stainless steel stub using conductive tape, followed by gold sputtering to avoid electrostatic charging of the membrane surface.

\subsection{RESULTS AND DISCUSSIONS}

\subsection{Influence of Polymer Formulation on the Membrane Morphology}

Table 2 summarizes the hollow fiber dimensions. It was observed that the membrane thickness slightly increased with the addition cSMM. This may be due to the thermodynamic compositional change which influences the diffusion of coagulant. Most likely the diffusion rate increased slightly in the ternary system compared to the binary system.

The area deformation ratio (ratio of spinneret $\left(A_{s}\right)$ to fiber $\left(A_{h f}\right)$ cross section area) for the fabricated membranes PES and PES-HBS were 0.76 and 0.83 respectively. This suggests a reduction in hollow fiber surface area when exiting the spinneret due to suppression and extrusion shear at spinneret wall and elongation from gravity at dry gap. However, as shown in Table 2, the thickness ratio of the fabricated membranes was nearly equal to 0.5 , which has been considered as good hollow fiber membrane strength properties [18].

The cross-sections of the three types of membranes are shown in Figure 1. The FESEM micrographs exhibited typical asymmetric structures with micro-pores and finger-like structures that acted as a micro-porous mechanical support. Particularly, approximate measured via FESEM pictograph, the asymmetric membranes showed top skin dense outer layers with thicknesses of 90-95 $\mu \mathrm{m}$ and 95-112 $\mu \mathrm{m}$ for unmodified PES and PES-HBS hollow fibers, respectively. Beneath the top skin layer, a porous sub-layer which is present in the form of spongelike, finger-like and macro-voids structures. Asymmetric structures that comprise of finger- 
Table 2 Dimension of the fabricated hollow fiber membranes

\begin{tabular}{llccc}
\hline Membrane ID & Membrane formulation & $\left.\begin{array}{c}\text { Membrane } \\
\text { thickness } \\
{[(\mathbf{O D}-\mathrm{ID}) / 2]}\end{array}\right]$ & $\begin{array}{c}\text { Thickness } \\
\text { ratio } \\
(\text { ID:OD) }\end{array}$ & $\begin{array}{c}\text { Deformation } \\
\text { area ratio } \\
{\left[\mathbf{A}_{\mathbf{s}}: \mathbf{A}_{\mathbf{h}} \mathbf{f}\right]}\end{array}$ \\
\hline PES & PES/NMP & 118.75 & 0.57 & 0.76 \\
PES-HBS & PES/PEG-HBS/NMP & 127.68 & 0.49 & 0.83 \\
\hline
\end{tabular}
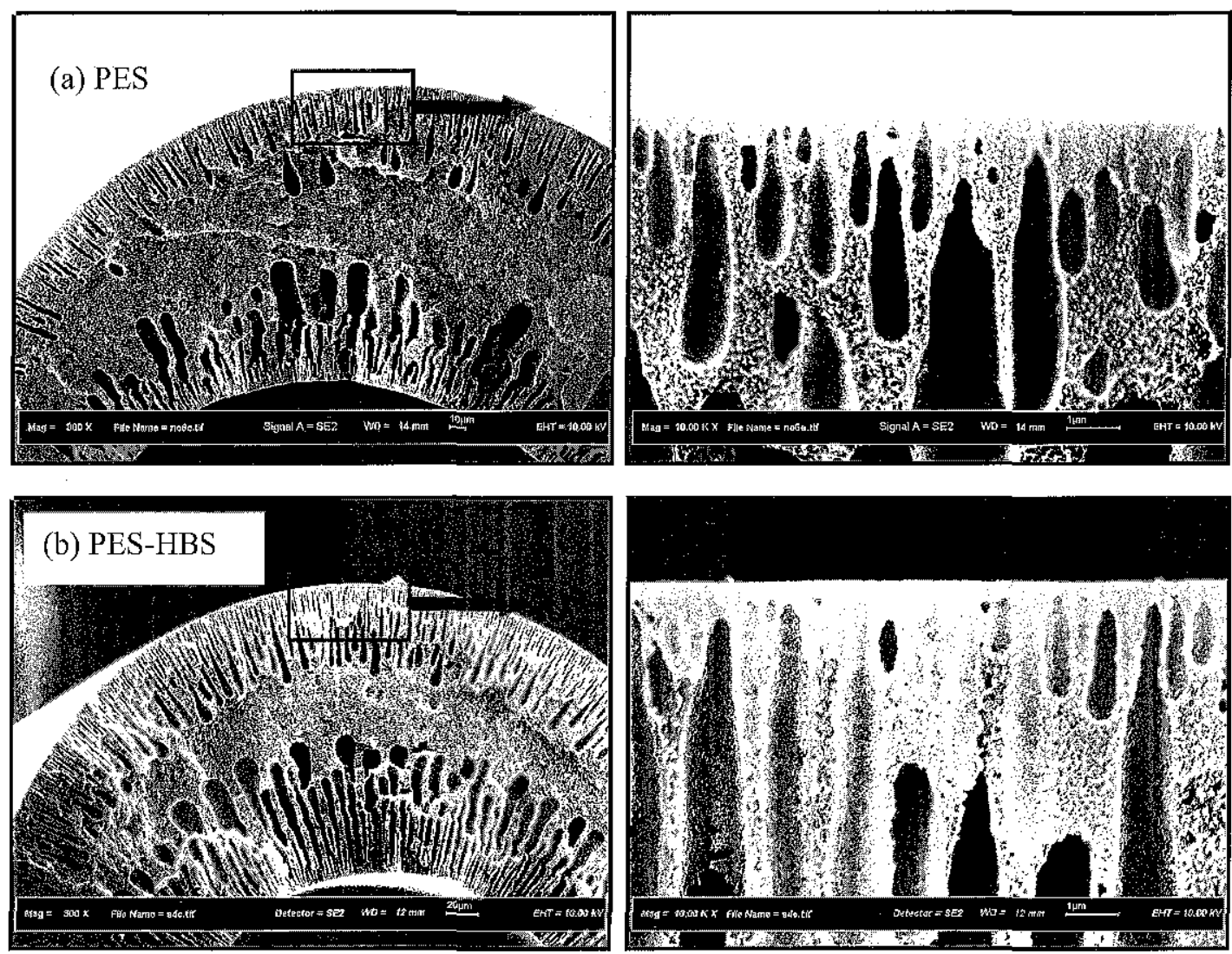

Figure 2 SEM cross section of hollow fiber membranes at magnification $300 \times$ and $10 \mathrm{~K} \times$

like structure were formed, signifying a rapid coagulation process when strong non-solvent such as water is used as a coagulant for the internal bore fluid and for the external coagulant.

In this study, the hollow fiber membranes were spun in a dry-wet condition, at $5 \mathrm{~cm}$ air gap. The dope compositions took different paths at the inner and outer surface, i.e. outward flow of the inner bore fluid while hollow fiber was traveling through the air-gap and inward coagulation fluid flow when the fiber reached the coagulation bath. Vigorous coagulation process initially occurred from the bore side, which caused instantaneous phase separation and froze shear induced orientation at the lumen $[17,19]$. This may suggest longer and larger finger-like micro-pores from the inner surface. Chung and Xudong [20] suggested that finger-like voids were created by the interconnection of spinodal decomposition with the aid of unbalanced localized stresses from 
surface tension, solvent/coagulant agglomeration, volumetric change, and radially convective flows of the inner and outer coagulants. Then, rapid solvent exchange of the dope solution continued and took place from its external surface towards the inner fiber when the nascent fiber entered into the coagulation bath. This phenomenon produced multilayered finger-like structure shown in the FESEM figure.

It seems that the general morphology of PES membranes is not affected very much by the addition of cSMM. There was only a small amount of CSMM added, which by comparison was only 0.05 ratio to the base polymer. However, an increase of dense skin top layer with the addition of cSMMs suggests functional modification due to the cSMM blending. This might be related to the migration of CSMMs to the surface during phase inversion in the dry-wet process. It can be attributed to thermodynamics principles as SMMs migrate to the surface to reduce its interfacial energy $[2,12-13,21-23]$.

\subsection{Energy Dispersive X-Ray Analysis}

Energy dispersive x-ray (EDX) of elemental analysis for the hollow fiber membranes shows the chemical composition for element carbon (C), oxygen (O), sulfur (S) and sodium (Na). The chemical compositions at the outer surface for the three types of membranes are summarized in Table 3. The addition of cSMM gave rise to the element of sodium in both modified PES samples. The amount of sodium was small $(<1 \%)$, but it reflects the occurrence of sodium salts that come from the end capping of the cSMM. Additionally, the amount of sulfur and oxygen has also increased approximately by $40 \%$ and $10 \%$ respectively for the modified PES membranes. This suggests an existence of sulfonate ion at the membrane surface, which contributed from the HBS.

\subsection{Effects of SMM Blending on Membrane Flux and Rejection}

The results of filtration performance are shown in Figure 3(a) for pressure normalized pure water permeation (PWP) flux and in 3 (b) for sodium chloride rejection at 6.89 Bar. As shown, the pure water fluxes of cSMM blended membranes are significantly higher than the unblended membrane at different operating pressures, i.e. the normalized pure water fluxes for PES and PESHBS were $2.1,4.5 \mathrm{~L} / \mathrm{m}^{2}$.h. Bar, respectively. The PWP was measured from the slope of the graph. The PWP result shows that CSMM blending to the casting solution has doubled the normalized water permeation flux of the hollow fiber membranes for this particular membrane preparation.

It is found from Figure 3(a) that the order of pure water permeation rate is; PES-HBS $>$ PES. According to the trade-off effect of flux and solutes rejection, the $\mathrm{NaCl}$ rejection performance would be in the opposite order. It was indeed so, however, the rejection of PES-HBS membrane

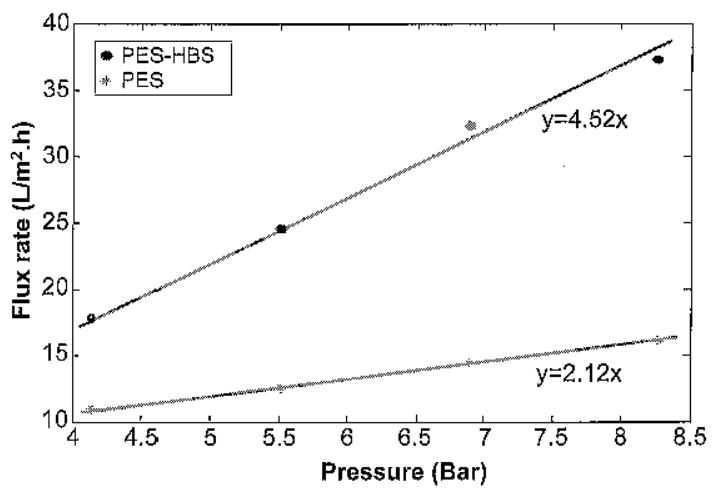

(a)

Table 3 elemental weight percent at the membrane outer surface by EDX analysis

\begin{tabular}{lcccc}
\hline Membrane ID & \multicolumn{4}{c}{ Elemental weight percentage at HF surface (\%) } \\
& Carbon (C) & Oxygen (O) & Sulfur (S) & Sodium (Na) \\
\hline PES & 73.0 & 17.8 & 9.2 & $\sim$ \\
PES-HBS & 66.4 & 19.5 & 13.3 & 0.8 \\
\hline
\end{tabular}




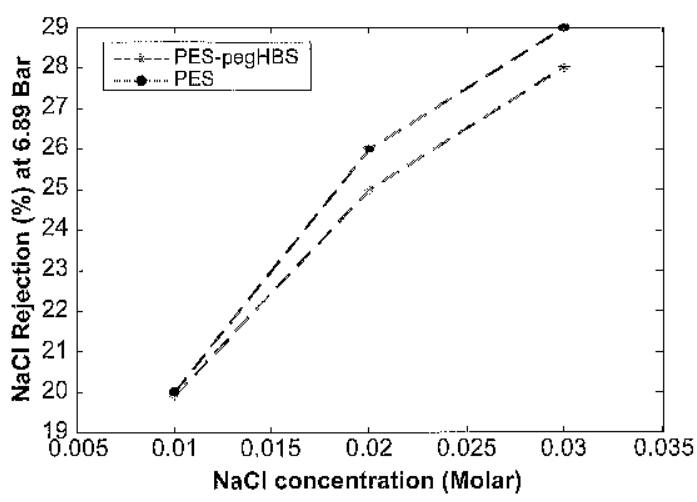

Figure 3 Filtration performance of membranes: (a) pure water permeation and (b) Sodium Chloride rejection at 100 psig at increasing concentration

was nearly as good as PES. Hence, it can be concluded that PES-HBS membrane has a superior nanofiltration performance. This probably is due to the introduction of the surface charge by blending ion carrying cSMM in the modified PES hollow fiber.

\subsection{CONCLUSIONS}

The first attempted PES hollow fiber blended with charged surface modifying macromolecules (cSMM) was successfully fabricated. The characterization of the membranes and the subsequent nano filtration experiments led to the following conclusions:

(1) A minimal morphology changes were found by the addition of $1 \%$ cSMM in PES membranes and yet a significant increment was observed in the thickness of the dense outer layer, which in turn increased the ionic rejection, especially for the case of PES-HBS.

(2) A preliminary investigation on membrane surface via EDX has revealed miscibility and the occurrence of cSMM at the membrane surface from the observation of sodium and sulfur. Yet, further confirmation by XPS analysis would provide better information of the elemental properties of the membranes.
(3) Modified PES by blending technique has successfully increased the permeation rate twice as much as the unblended membrane.

(4) CSMM with PES was found to be capable to create better performance of membranes especially for ionic separation. However, more understanding on CSMM migration to surface is desired. As well, the capability for cSMM to enhance the membrane surface charge, especially for hollow fiber membranes, needs to be further studied.

\section{ACKNOWLEDGEMENT}

The authors would like to acknowledge the financial support from of the Ministry of Higher Education (MOHE) grant and Universiti Malaysia Sabah (UMS). The author is thankful to the Universiti Teknologi Malaysia (UTM) for providing excellent research environment to complete this work.

\section{REFERENCES}

[1] Thomas, G. 1991. Advances in Water Treatment and Environment Management. Spon Press.

[2] Rana, D., T. Matsuura, R.M. Narbaitz, and C. Feng. 2005. Development and Characterization of Novel Hydrophilic Surface Modifying Macromolecule for Polymeric Membranes. J. of Membr. Sci. 249: 103-112.

[3] Mulder, M. 2002. Basic Principles of Membrane Technology. Kluwer Academic Publishers: Netherlands.

[4] Rachwal, A.J., J. Khow, J.S. Colborne, and J. O'Donnel. 1994. Water Treatment for Public Supply in the 1990's - A Role For Membrane Technology? Desalination. 427-436.

[5] Cartwright, P.S. 1992. Industrial Wastewater Treatment with Membranes - US Perspective. Water Sci. Tech. 25(10): 373390.

[6] Du, R., and J. Zhao. 2003. Positively Charged Composite Nanofiltration Membrane Prepared by Poly(N,N-dimethyla- 
minoethyl methacrylate)/polysulfone. $/$. of Applied Polymer Sci. 91: 2721-2728.

[7] Castilho, L.R., W.D. Deckwer, and F.B Anspach. 2000. Influence of Matrix Activation and Polymer Coating on the Purification of Human IgG with Protein A Affinity Membranes. J. Membr. Sci. 72: 269-277.

[8] Brink, L.E.S., S.J.G. Elbers, T. Robbertsen, and P. Both. 1993. The Anti-fouling Action of Polymers Preadsorbed on Ultrafiltration and Microfiltration Membranes. J. Membr. Sci. 76: 281-291.

[9] Ulbricht, M. 2006. Advanced Functional Polymer Membranes. Polymer. 47: 22172262.

[10] Hamza, A., V.A. Pham, T. Matsuura, and J.P. Santerre. 1997. Development of Membranes with Low Surface Energy to Reduce that Fouling in Ultrafiltration Applications. Journal Membrane of Science. 131: 217227.

[11] Khayet, M., D.E. Sul, R.M. Narbaitz, J.P. Santerre, and T. Matsuura. 2003. Study on Surface Modification by Surface-modifying Macromolecules and its Applications in Membrane-separation Processes. J. of Applied Polymer Science. 89(11): 29022916.

[12] Suk, D.E., T. Matsuura, H.B. Park, and Y.M. Lee. 2006. Synthesis of a New Type of Surface Modifying Macromolecules (nSMM) and Characterization and Testing of nSMM Blended Membranes for Membrane Distillation. J. of Membr. Sci. 277: 177-185.

[13] Pham, V.A., J.P. Santerre, T. Matsuura, and R.M. Narbaitz. 1998. Application of Surface Modifying Macromolecules on Polyethersulfone Membranes: Influence on PES Surface Chemistry and Physical Properties. Journal of Applied Polymer Sci. 73: 1363-1378.

[14] Mosqueda-Jimenez, D.B., R.M. Narbaitz, T. Matsuura, G. Chowdhury, G. Pleizier, and J.P. Santerre. 2004. Influence of Processing Conditions on the Properties of Ultrafiltration Membranes. J. of Membr. Sci. 231: 209-224.

[15] Ismail, A.F., and Hassan, A.R. 2006. Formation and Characterization of Asymmetric Nanofiltration Membrane: Effect of Shear
Rate and Polymer Concentration. $J$. of Membr. Sci. 270: 57-72.

[16] Fang, Y., V.A. Pham, T. Matsuura, J.P Santerre, and R.M. Narbaitz. 1994. Effect of Surface-modifying Macromolecules and Solvent Evaporation Time on the Performance of Polyethersulfone Membranes for the Separation of Chloroform/water Mixtures by Pervaporation. J. Appl. Polym. Sci. 54(12): 1937-1943.

[17] Ismail, A.F, M.I. Mustaffar, R.M. Illias, and M.S. Abdullah. 2006. Effect of Dope Extrusion Rate on Morphology and Performance of Hollow Fibre Membranes for Ultrafiltration. Sep. \& Purification Tech. 49: 10-19.

[18] Wood, H., J. Wang, and S. Sourirajan. 1993. The Effect of Polyethersulfone Concentration on Flat and Hollow Fiber Membrane Performance. Sep. Sci. Technol. 28: 22972318.

[19] Qin, J.J., Y.M. Cao, and M.H. Oo. 2006. Preparation of Polyethersulfone Hollow Fiber Ultrafiltration Membranes for Removal of NOM. $J$, of Applied Polymer Sci. 99: 430-435.

[20] Chung, T.S., and H. Xudong. 1997. Effect of Air Gap Distance on the Morphology and Thermal Properties of Polyethersulfone Hollow Fiber. Journal of Applied Polymer Sci. 66: 1067-1077.

[21] Mosqueda-Jimenez, D.B, R.M. Narbaitz, and T. Matsuura. 2004. Manufacturing Conditions of Surface-modified Membranes: Effects on Ultrafiltration Performance. Separation and Purification Technology. 37: 51-67.

[22] Mosqueda-Jimenez, D.B, R.M. Narbaitz, and T. Matsuura. 2006. Effects of Preparation Conditions on the Surface Modification and Performance of Polyethersulfone Ultrafiltration Membranes. J. of Applied Polymer Sci. 99: 2978-2988.

[23] Khayet, M., J. Mengual, and T. Matsuura. 2005. Porous Hydrophobic/hydrophilic Composite Membranes Application I Desalination using Direct Contact Membrane Distillation. J. of Membr. Sci. 252: 101-113. 